\title{
Compósitos de fibra de coco/LDPE: efeito do tratamento superficial das fibras de coco em compósitos verdes
}

\section{Coconut fiber/LDPE composites: effect of surface treatment of coconut fibers to produced green composites}

\author{
Yves Nicolau Wearn ${ }^{1}$, Larissa Stieven Montagna ${ }^{1}$,
} Fábio Roberto Passador ${ }^{1}$

\author{
${ }^{1}$ Laboratório de Tecnologia de Polímeros e Biopolímeros (TecPBio), Universidade Federal de São Paulo, Rua Talim 330 , \\ CEP 12231-280, São José dos Campos, São Paulo, Brasil. \\ e-mail: fabio.passador@gmail.com
}

\section{RESUMO}

O desenvolvimento de compósitos poliméricos reforçados com fibras naturais tem recebido grande destaque como tecnologia alternativa para o processamento de novos materiais que proporcionem um menor impacto ambiental, associado a uma baixa densidade, biodegradabilidade e propriedades mecânicas interessantes. Fibras naturais provenientes do coco verde apresentam, devido à sua composição química, maior rigidez e resistência mecânica comparada a outras fibras vegetais. Porém, fibras vegetais possuem uma baixa interação com a matriz polimérica, em virtude de seu caráter hidrofílico quando comparado à matriz polimérica, hidrofóbica. Neste trabalho, fibras de coco (FC) foram tratadas quimicamente por extração alcalina com hidróxido de sódio $(\mathrm{NaOH})$ e mecanicamente utilizando uma ponteira ultrassônica em meio aquoso, e a técnica para averiguar os efeitos dos tratamentos nas fibras foi a microscopia eletrônica de varredura (MEV). Compósitos de polietileno de baixa densidade (LDPE) com diferentes teores de fibra de coco $(5$ e $10 \% \mathrm{~m} / \mathrm{m}$ ) foram preparados por mistura no estado fundido, utilizando um homogeneizador de alta rotação (DRAIS), seguido por prensagem a quente e estampagem para preparação de corpos de prova. Os compósitos de LDPE/FC foram caracterizados quanto às propriedades térmicas (calorimetria exploratória diferencial - DSC), mecânicas (ensaio de tração uniaxial e ensaio de dureza), características morfológicas (MEV) e de superfície (teste de ângulo de contato). $\mathrm{O}$ teor de fibra em massa exerceu influência nas propriedades mecânicas, de forma que um aumento do teor de fibra de coco em massa reduz ligeiramente a resistência à tração e provoca um aumento em torno de $100 \%$ no valor do módulo elástico do compósito. Por meio dos resultados obtidos por MEV e ângulo de contato, ambos os tratamentos se mostraram eficientes, porém sugerindo melhor eficiência no tratamento via extração alcalina.

Palavras-chave: Compósitos poliméricos, fibra de coco; polietileno de baixa densidade, tratamentos superficiais.

\section{ABSTRACT}

The development of polymer composites reinforced with natural fibers have gained attention as alternative technology for the processing of new material that provide a lower environmental impact, associated with lower density, biodegradability and interesting mechanical properties. Natural fibers that come from the green coconut have higher stiffness and mechanical resistance when compared with other natural fibers, considering its chemical composition. Although, natural fibers show a weak interaction with the polymer matrix, due to its hydrophilicity when compared to the matrix, hydrophobic. In this work, coconut fibers (CF) were chemically treated by alkalization with sodium hydroxide $(\mathrm{NaOH})$ and mechanically treated by ultrasound in aqueous media. Low-density polyethylene (LDPE)/CF composites were prepared with different contents of coconut fiber ( 5 and $10 \mathrm{wt} \%$ ), in the molten state using a homogenizer with high rotation (DRAIS) and stamping for the preparation of the specimens. The treated fibers were characterized by scanning electron microscopy (SEM) and the LDPE/FC composites were characterized according to the thermal properties (differential scanning calorimetry - DSC), mechanical properties (tensile tests) and morphology (SEM). From the 
results obtained, it can be concluded that no significant change was observed with the different treatments for composites with $5 \mathrm{wt} \%$ of CF compared to the neat LDPE. The best results of mechanical resistance noticed on composites with $5 \mathrm{wt} \%$ of $\mathrm{CF}$ when compared with composites with $10 \mathrm{wt} \%$ of $\mathrm{CF}$. The ultrasound treatment demonstrated the best performance. The crystallization degree of the neat LDPE was not affected by the presence of the coconut fibers, although there was a good interaction and adhesion between the CF and the polymer matrix.

Keywords: Polymer composites, coconut fiber, low-density polyethylene, superficial treatments.

\section{INTRODUÇÃO}

A fibra de coco, proveniente da casca do coco verde, é uma das fibras naturais mais empregadas atualmente, como alternativa ao desenvolvimento de tecnologias que gerem um menor impacto ambiental. Quando comparada a outras fibras naturais, como a juta, o sisal e a fibra de banana, destaca-se por possuir um maior percentual de lignina em sua composição, componente que proporciona uma maior rigidez e resistência à fibra [1-3]. A Figura 1 identifica as partes do fruto, formado a partir da drupa, uma semente composta pelo epicarpo (camada mais externa fina e lisa); o mesocarpo (camada intermediária mais espessa e fibrosa, envolvido pelo epicarpo); e o endocarpo (camada lenhosa e dura). A fibra pode ser extraída tanto do mesocarpo quanto do epicarpo, e o peso médio do fruto varia de 3 a $4 \mathrm{~kg}$ [4-6].

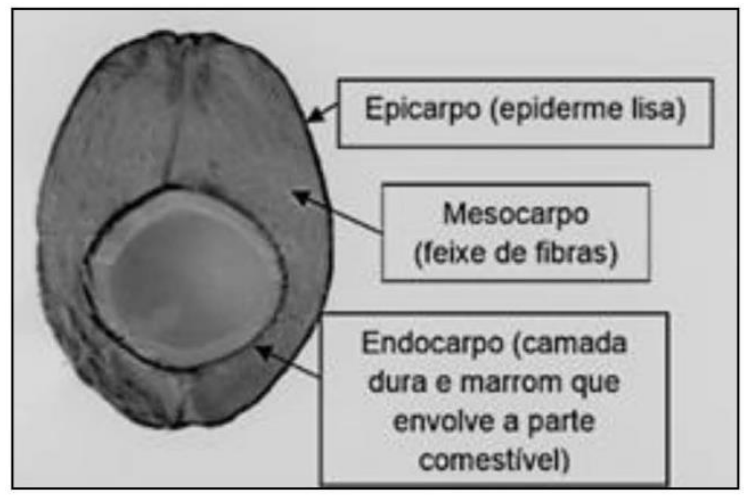

Figura 1: Ilustração do corte longitudinal do fruto do coco verde com a descrição de suas partes. Adaptado de [7], 2018.

Esse tipo de material pode ser utilizado na fabricação de tapetes e capachos, cordame especial para navios, escovas e vassouras, enchimento de almofadas, estofamento de automóveis, entre outros. Em materiais compósitos, a fibra pode ser empregada como agente de reforço em polímeros, como o poliéster, o polipropileno, o polietileno e polímeros biodegradáveis. Além de reduzir a quantidade de resíduos emitidos, é um processo natural e renovável, e possui um custo mais baixo. [4-5, 8-9].

Os impactos ambientais que decorrem da deposição de resíduos do fruto são um importante aspecto a ser levado em consideração, visto que seu descarte provoca, dentre outros fatores, a necessidade de uma maior demanda de área para que os resíduos sejam descartados, bem como poluição visual, a proliferação de vetores e a emissão de gases poluentes devido à sua decomposição, como o gás metano, e a contaminação do solo [10-11]. Segundo estimativas, 6,7 milhões de toneladas de casca são descartadas por ano em aterros sanitários [12]. Em cidades litorâneas, como Fortaleza, 70\% do lixo depositado nas orlas das praias corresponde aos resíduos gerados pelo descarte do fruto [11].

O polietileno de baixa densidade (LDPE) - um dos polímeros termoplásticos mais utilizados no mundo - é processado a altas temperaturas (entre 100 e $300^{\circ} \mathrm{C}$ ) e a altas pressões (entre 1000 e $3000 \mathrm{~atm}$ ), e é parcialmente cristalino (50 a 60\%), com temperatura de fusão cristalina (Tm) entre 110 e $155^{\circ} \mathrm{C}$. Destacase por apresentar alta tenacidade, alta resistência ao impacto, boa processabilidade e estabilidade, além de não ser tóxico e possuir baixa absorção de umidade. Suas aplicações incluem filmes em embalagens de alimentos, industriais e agrícolas, e revestimentos de mangueiras, tubos, fios e cabos [13].

Compósitos poliméricos reforçados por fibras curtas - com razão de aspecto (comprimento/largura) inferior a 10 - destacam-se por apresentar baixo peso e alta resistência mecânica, além de baixo custo de produção, permitindo uma maior acessibilidade desse tipo de material. O diâmetro, o comprimento, a fração volumétrica, o arranjo e a distribuição na matriz polimérica são alguns dos aspectos das fibras que influenciam diretamente nas propriedades finais do compósito, associado ao grau de interação entre o polímero e a fibra por meio de sua interface [14]. Amuthakkannan et at. [14] analisaram os efeitos que o tamanho e os teores de fibras curtas de basalto implicam nas propriedades mecânicas de compósitos poliméricos e identifi- 
caram, por meio de ensaios de tração, melhores resultados sob tamanho ótimo de fibra de $10 \mathrm{~mm}$ e a um teor ótimo de $68 \%$ de dispersão na matriz.

No que diz respeito às fibras naturais como agente de reforço em compósitos poliméricos, um importante aspecto deve ser considerado. A matriz é predominantemente hidrofóbica, enquanto que as fibras possuem caráter hidrofílico. Tais características tornam necessária a aplicação de tratamentos - físicos, químicos ou mecânicos - que aumentem a eficiência do material e reduzam o grau de hidrofilicidade das fibras, visto que ocorre uma baixa adesão da interface matriz/reforço no compósito $[10,15]$.

O tratamento superficial químico mais empregado em fibras vegetais é a extração alcalina, por solução de hidróxido de sódio $(\mathrm{NaOH})$, que consiste na agitação seguida de lavagem das fibras imersas em solução aquosa de $\mathrm{NaOH}$. Esse beneficiamento remove alguns dos componentes da superfície da fibra, como a hemicelulose e a lignina, aumentando a aderência entre o reforço e a matriz, sem prejudicar as propriedades naturais das fibras. O esquema reacional que ocorre durante o tratamento está representado na Figura 2 [16].

Fibra-OH $+\mathrm{NaOH} \rightarrow$ Fibra-O-Na ${ }^{+}+\mathrm{H}_{2} \mathrm{O}$

Figura 2: Esquema reacional das fibras com o hidróxido de sódio.

A remoção da lignina, presente em grande quantidade na fibra, não apenas aumenta a adesão interfacial como também reduz a transferência de carga entre a fibra e a matriz polimérica. Durante esse processo, ocorre a degradação do éster e das cadeias glicosídicas da estrutura molecular da lignina, modificando sua estrutura química. Esse processo aumenta a rugosidade de sua superfície, aprimorando a razão de aspecto e proporcionando melhores propriedades mecânicas ao compósito polimérico [17-18].

Tal efeito foi observado por Nam et al. [19], que analisaram a influência do tratamento alcalino nas propriedades mecânicas e de interface de poli(butileno succionato) reforçado com fibras de coco, por meio de uma solução aquosa de $5 \%$ de $\mathrm{NaOH}$ a quatro intervalos de absorção (24h, 48h, 72h e 96h) a temperatura ambiente, e secagem por 2 dias. Foi observado, após a realização de ensaios de tração das amostras, um aumento da resistência mecânica à medida que aumentava o tempo de absorção, até um período ótimo de 72 horas. Novos testes foram feitos, sob 3\%, 5\% e 7\% de solução aquosa de $\mathrm{NaOH}$, durante as mesmas 72 horas, indicando que o teor de solução empregada também exerce influência nas propriedades finais, visto que sob $5 \%$ de solução as propriedades mecânicas em tração foram as superiores.

Esmeraldo [8], submeteu fibras de coco sob tratamento via extração alcalina a concentrações em solução aquosa de $5 \%$ e $10 \%$ de $\mathrm{NaOH}$ em matrizes fenólicas de líquido de casca de castanha de caju (LCC). Notou-se um resultado mais satisfatório nas amostras com fibras de coco a $10 \%$ de $\mathrm{NaOH}$, com uma superfície de fibra mais rugosa, conforme também pode ser observado nas micrografias dos compósitos por meio de uma melhor interação reforço/matriz. O experimento também foi realizado com fibras de juta, e comparativamente, houve um melhor desempenho mecânico sob tração a 5\% de $\mathrm{NaOH}$ em compósitos reforçados pelas fibras de juta, e sob $10 \%$ de $\mathrm{NaOH}$ nos compósitos de fibras de coco.

Hussain et al. [20] estudaram o efeito de diferentes comprimentos e teores de fibra de coco em massa tratadas com $\mathrm{H}_{2} \mathrm{O}_{2}$ e NaOH em compósitos de polietileno de alta densidade (HDPE), avaliando as propriedades mecânicas. Os resultados indicaram um aumento da resistência em tração e uma redução na resistência em flexão conforme maior a fração volumétrica de fibra de coco distribuída na matriz, e uma variação mais significativa dos efeitos dos comprimentos de fibra apenas nos ensaios de flexão e impacto.

Além de tratamentos químicos, também podem ser empregados em fibras vegetais tratamentos fisicos, como o ultrassom. Tal procedimento modifica a superfície, morfologia e composição das fibras por meio das colisões que ocorrem entre o líquido no qual as fibras ficam mergulhadas e a superfície sólida das mesmas [21]. Santos et al. [22] avaliaram a influência do tratamento por plasma a frio de $\mathrm{N}_{2}$ e o tratamento químico com solução de $\mathrm{NaOH}$ em fibras de curauá como reforço em compósitos de Poliamida-6 (PA6), e ambos os processos se mostraram eficientes. O tratamento com plasma aumentou a resistência mecânica e o módulo elástico sob tração dos compósitos, e a extração alcalina melhorou a resistência e o módulo elástico tanto em tração como em flexão para as amostras. Ainda assim, tratamentos como o tratamento frio por plasma e o tratamento corona, embora não prejudiquem o meio ambiente, possuem um custo bastante elevado [16].

Neste trabalho, o efeito de diferentes tratamentos superficiais em fibras de coco - a extração alcalina e o tratamento com ponteira ultrassônica em meio aquoso - foi avaliado no desenvolvimento de compósitos poliméricos de LDPE reforçados com fibras de coco. Um grande diferencial desse trabalho consiste na técnica de processamento utilizada para a produção dos compósitos. O processamento dos materiais foi realizado através de homogeneizador rotacional (termocinético), em que a fusão do componente polimérico ocorre por aquecimento viscoso gerado por atrito do rotor de alta rotação $(3000 \mathrm{rpm})$. A validação da eficácia dos trata- 
mentos superficiais das fibras de coco e do processamento utilizado foi realizado por meios da avaliação das propriedades térmicas, mecânicas e características morfológicas do compósito produzido sob os diferentes tratamentos utilizados e comparação com compósitos preparados com fibras de coco in natura.

\section{MATERIAIS E MÉTODOS}

Para o desenvolvimento desse trabalho foram utilizados:

- Fibras de coco in natura, fornecidas pela empresa Coco Verde/RJ (Rio de Janeiro-RJ/Brasil);

- Polietileno de baixa densidade (LDPE), especificação LD5000A, fornecido pela Braskem, com massa específica de $0,921 \mathrm{~g} / \mathrm{cm}^{3}$ e índice de fluidez de $1,4 \mathrm{~g} / 10 \min \left(190{ }^{\circ} \mathrm{C} / 2,16 \mathrm{~kg}\right)$.

\subsection{Tratamentos das fibras de coco}

As fibras de coco foram cortadas, com o auxílio de uma cortadeira (Samos) em comprimentos de aproximadamente $10 \mathrm{~mm}$ e posteriormente submetidas a dois tipos de tratamentos superficiais, o tratamento alcalino (extração alcalina) com solução aquosa de hidróxido de sólido $(\mathrm{NaOH})$ e processador ultrassônico em meio aquoso.

\subsubsection{Tratamento alcalino (extração alcalina) com solução aquosa de $\mathrm{NaOH}$}

O tratamento químico alcalino realizado nas fibras de coco in natura consistiu na imersão de $90 \mathrm{~g}$ da fibra em uma solução aquosa de $\mathrm{NaOH}(10 \% \mathrm{~m} / \mathrm{m})$, sob agitação magnética (Corning modelo PC-420D) por uma hora. Em seguida, foi feita a lavagem abundante das fibras com água destilada até atingir pH neutro. Após a completa neutralização da solução, as fibras de coco foram acondicionadas em uma estufa a $60{ }^{\circ} \mathrm{C}$ por um período de 24 horas para secagem.

\subsubsection{Processador ultrassônico em meio aquoso}

As fibras de coco in natura foram tratadas em um processador ultrassônico (Sonics Vibra Cell, modelo VC 750) com frequência de $20 \mathrm{~Hz}$ em água, sem a utilização de reagentes químicos que agridam tanto a fibra como o meio ambiente. Foram imersas $30 \mathrm{~g}$ de fibra em água em proporção de $5 \% \mathrm{~m} / \mathrm{m}$. O tratamento foi realizado durante um período de 45 minutos, com movimentação a cada 15 minutos. Em seguida, as fibras de coco foram colocadas em uma estufa a $60{ }^{\circ} \mathrm{C}$ para secagem durante 24 horas.

\subsection{Caracterização morfológica dos compósitos de LDPE/fibra de coco}

A caracterização das fibras antes e após os tratamento foi realizada por meio da microscopia eletrônica de varredura (MEV), que permite a caracterização morfológica da fibra de coco pós-tratamentos. A análise foi feita em um microscópio eletrônico de varredura (Inspect S50 - FEI Company ${ }^{\circledR}$ ), operando a $15 \mathrm{keV}$. As amostras de fibras foram fixadas em um $s t u b$ e metalizadas com ouro.

\subsection{Processamento dos compósitos de LDPE/fibra de coco}

Os compósitos de LDPE foram preparados com teores de 5 e $10 \% \mathrm{~m} / \mathrm{m}$ de fibra de coco e foram obtidos por meio da mistura dos componentes no estado fundido. Assim, foi utilizado um homogeneizador rotacional termocinético do tipo DRAIS (MH Instrumentos, modelo MH-50H) com rotação de 3000 rpm, no qual a fusão do polímero e a mistura e homogeneização com as fibras de coco são produzidas por meio do aquecimento viscoso devido ao atrito gerado entre o material polimérico e o rotor. Posteriormente, o material fundido foi prensado a quente utilizando uma prensa hidropneumática (MH Equipamentos, modelo PR8H) a $190{ }^{\circ} \mathrm{C}$, por 3 minutos e 5 bar de pressão, produzindo placas com 3,2 $\mathrm{mm}$ de espessura. Após a preparação das placas, os corpos de prova foram estampados utilizando uma estampadeira pneumática da CEASTInstron, sendo obtidos corpos de prova para aplicação de ensaios térmicos, mecânicos e morfológicos. A Tabela 1 apresenta a identificação dos materiais estudados, sendo as amostras A1 correspondentes ao compósito de LDPE com fibra de coco não tratada, as amostras A2 são de compósitos de LDPE com fibra de coco tratada via extração alcalina e A3 representa as amostras produzidas com fibra de coco tratada via ponteira ultrassônica. 
Tabela 1: Identificação dos compósitos conforme o tratamento aplicado.

\begin{tabular}{c|c}
\hline IDENTIFICAÇÃO & AMOSTRA \\
\hline $\mathrm{A} 1-5$ & $\mathrm{LDPE} / 5 \% \mathrm{FC}$ (in natura) \\
\hline $\mathrm{A} 2-5$ & $\mathrm{LDPE} / 5 \% \mathrm{FC}(\mathrm{NaOH})$ \\
\hline $\mathrm{A} 3-5$ & $\mathrm{LDPE} / 5 \% \mathrm{FC}$ (ultrassom) \\
\hline $\mathrm{A} 1-10$ & $\mathrm{LDPE} / 10 \% \mathrm{FC}$ (in natura) \\
\hline $\mathrm{A} 2-10$ & $\mathrm{LDPE} / 10 \% \mathrm{FC}(\mathrm{NaOH})$ \\
\hline $\mathrm{A} 3-10$ & $\mathrm{LDPE} / 10 \% \mathrm{FC}$ (ultrassom) \\
\hline
\end{tabular}

\subsection{Caracterização térmica dos compósitos de LDPE/fibra de coco}

A caracterização térmica dos compósitos LDPE/fibra de coco foi feita por meio da análise de calorimetria exploratória diferencial (DSC), realizada em um equipamento da TA Instruments, modelo Q100. As amostras foram submetidas a um ciclo térmico de aquecimento a partir da temperatura ambiente até $200{ }^{\circ} \mathrm{C}$, com taxa de aquecimento de $10{ }^{\circ} \mathrm{C} / \mathrm{min}$, sob atmosfera de nitrogênio, seguido por um ciclo de resfriamento até temperatura ambiente com uma taxa de $10^{\circ} \mathrm{C} / \mathrm{min}$ e um segundo ciclo de aquecimento até $200^{\circ} \mathrm{C}$.

\subsection{Caracterização mecânica dos compósitos de LDPE/fibra de coco}

Para a caracterização mecânica foram realizados ensaio de tração uniaxial e ensaio de dureza.

\subsubsection{Ensaios de tração dos compósitos de LDPE/fibra de coco}

No ensaio de tração, foram empregados 5 corpos de prova para cada composição e foi utilizada uma máquina universal de ensaios de marca EMIC, modelo GR048, com velocidade de subida da travessa de 50 $\mathrm{mm} / \mathrm{min}$, e célula de carga de $50 \mathrm{kN}$.

\subsubsection{Ensaios de dureza dos compósitos de LDPE/fibra de coco}

Para o ensaio de dureza, utilizou-se um durômetro Shore D (Instrutherm - modelo DP-400). Os resultados foram obtidos a partir de uma média de 5 leituras na superfície do compósito em pontos espaçados entre si.

\subsection{Caracterização morfológica dos compósitos de LDPE/fibra de coco}

Os aspectos morfológicos das fibras de coco antes e após os diferentes tratamentos e dos compósitos poliméricos LDPE/fibra de coco produzidos foram determinados por meio da microscopia eletrônica de varredura $(\mathrm{MEV})$. A análise foi feita em um microscópio eletrônico de varredura (Inspect S50 - FEI Company ${ }^{\circledR}$ ), operando a $15 \mathrm{keV}$. As amostras dos compósitos de LDPE/FC foram criofraturadas, fixadas em stubs de alumínio e metalizadas com ouro.

\subsection{Caracterização de superfície dos compósitos de LDPE/fibra de coco}

A caracterização de superfície dos compósitos poliméricos, a fim de averiguar o grau de molhabilidade das amostras, foi feita através de um teste de ângulo de contato por meio de um goniômetro Ramé-Hart (modelo 500), no qual foram feitas as medidas dos ângulos utilizando como líquido, água destilada com o método da gota séssil $\left(1,0 \mu \mathrm{L}, 25^{\circ} \mathrm{C}\right)$. 


\section{RESULTADOS}

\subsection{Caracterização morfológica das fibras de coco tratatas}

A Figura 3 apresenta as micrografias das FC da região superficial das fibras de coco in natura e modificadas via diferentes tratamentos (solução de $\mathrm{NaOH}$ e em meio aquoso com ponteira ultrassônica).
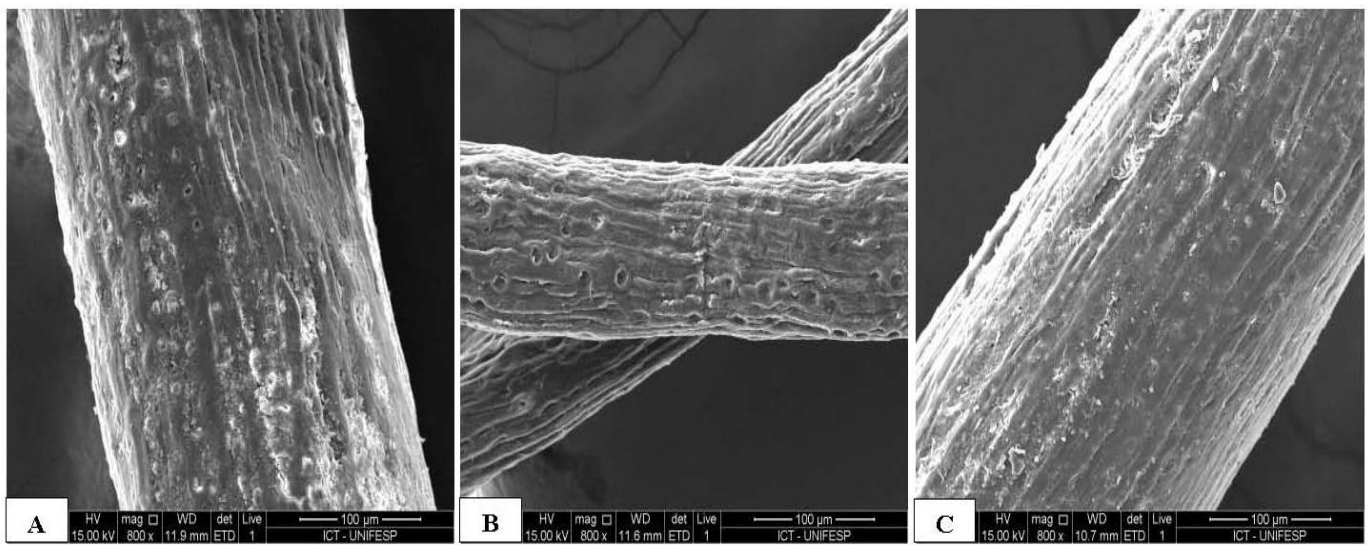

Figura 3: Micrografias de MEV das fibras de coco: (a) in natura (sem tratamento), (b) após tratamento com solução de $\mathrm{NaOH}$ e (c) após tratamento em meio aquoso e ponteira ultrassônica, com ampliação de 800x.

\subsection{Caracterização térmica dos compósitos LDPE/fibra de coco}

A Figura 4 apresenta os termogramas de DSC das amostras de LDPE puro e dos compósitos poliméricos estudados durante o primeiro aquecimento, o resfriamento e o segundo aquecimento. A Tabela 1 corresponde aos parâmetros térmicos obtidos.
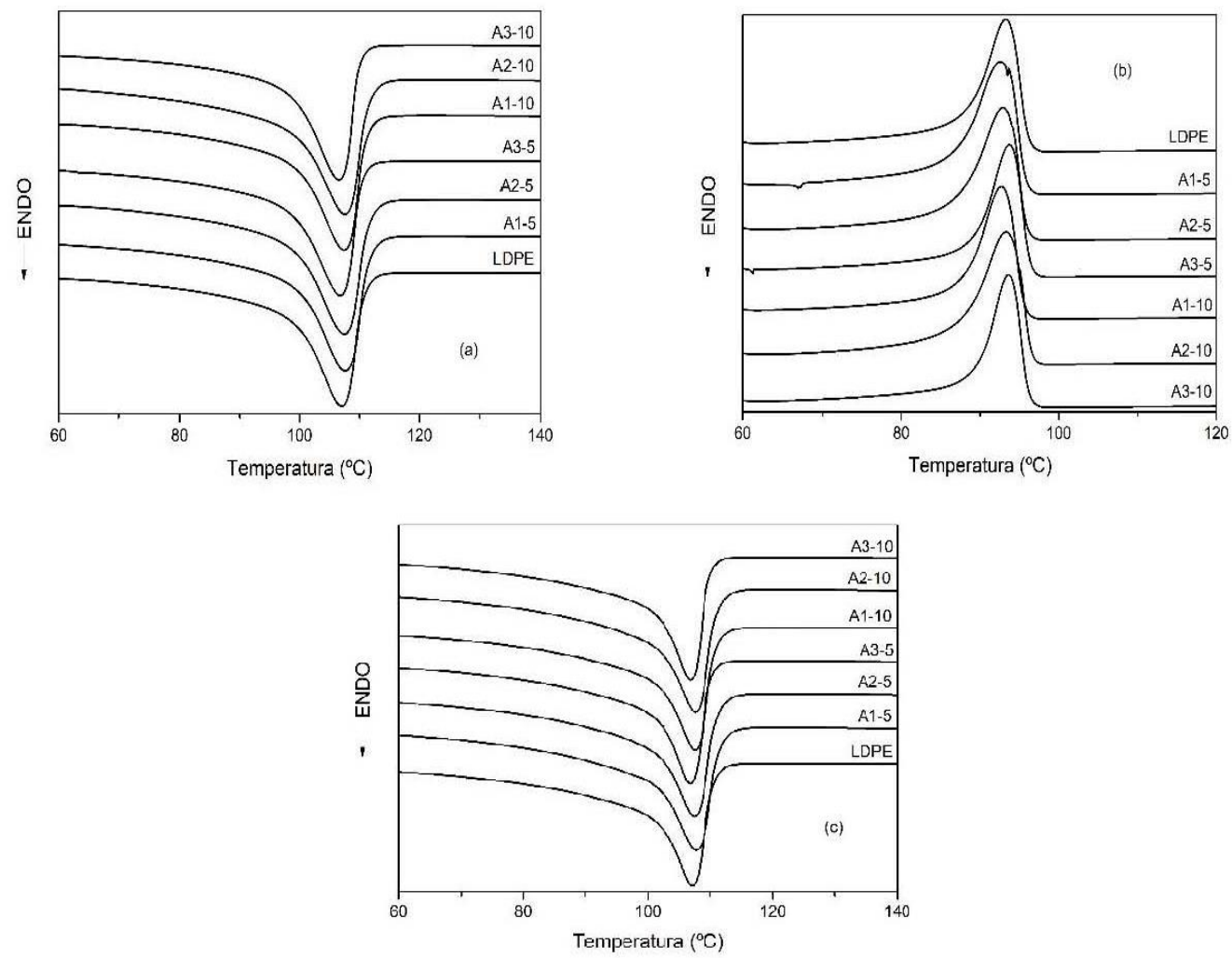

Figura 4: Termogramas de DSC dos compósitos A1, A2 e A3 com os diferentes teores de fibra de coco (a) durante o primeiro aquecimento, (b) durante o resfriamento e (c) durante o segundo aquecimento. 
A Tabela 2 apresenta os valores das propriedades térmicas obtidas por DSC das amostras de LDPE e dos compósitos LDPE/fibra de coco analisados conforme os diferentes tipos de tratamento aplicados (solução de $\mathrm{NaOH}$ e ultrassom) e os diferentes teores de fibra de coco no compósito polimérico (5 e $10 \% \mathrm{~m} / \mathrm{m})$.

Tabela 2: Propriedades térmicas dos compósitos poliméricos obtidas por DSC.

\begin{tabular}{c|c|c|c|c|c|c|c}
\hline AMOSTRAS & $\mathbf{T m}_{\mathbf{1}}\left({ }^{\circ} \mathbf{C}\right)$ & $\Delta \mathbf{H m}_{\mathbf{1}}(\mathbf{J} / \mathbf{g})$ & $\mathbf{X c}_{\mathbf{1}}(\%)$ & $\mathbf{T c}\left({ }^{\circ} \mathbf{C}\right)$ & $\mathbf{T m}_{\mathbf{2}}\left({ }^{\circ} \mathbf{C}\right)$ & $\Delta \mathbf{H m}_{\mathbf{2}}(\mathbf{J} / \mathbf{g})$ & $\mathbf{X c}_{\mathbf{2}}(\%)$ \\
\hline LDPE & 107 & 90,83 & 31,0 & 93 & 107 & 83,17 & 28,4 \\
\hline A1-5 & 108 & 82,56 & 29,7 & 93 & 108 & 81,42 & 29,3 \\
\hline A2-5 & 108 & 83,70 & 30,1 & 93 & 107 & 81,87 & 29,4 \\
\hline A3-5 & 107 & 79,36 & 28,5 & 94 & 107 & 79,25 & 28,5 \\
\hline A1-10 & 107 & 127,5 & 48,3 & 93 & 107 & 73,86 & 28,0 \\
\hline A2-10 & 108 & 80,37 & 30,5 & 93 & 107 & 72,72 & 27,6 \\
\hline A3-10 & 107 & 77,24 & 29,3 & 93 & 107 & 75,34 & 28,6 \\
\hline
\end{tabular}

Onde: $\mathrm{Tm}_{1}$ : temperatura de fusão cristalina medida no primeiro ciclo de aquecimento, $\Delta \mathrm{Hm}_{1}$ : energia de entalpia de fusão cristalina medida no primeiro ciclo de aquecimento, $\mathrm{Xc}_{1}$ : grau de cristalinidade calculado no primeiro aquecimento, $\mathrm{T}_{\mathrm{c}}$ : temperatura de cristalização medida no ciclo de resfriamento, $\mathrm{Tm}_{2}$ : temperatura de fusão cristalina medida no segundo ciclo de aquecimento, $\Delta \mathrm{Hm}_{2}$ : energia de entalpia de fusão cristalina medida no segundo ciclo de aquecimento e $\mathrm{Xc}_{2}$ : grau de cristalinidade calculado no segundo aquecimento.

O grau de cristalinidade dos polímeros foi calculado utilizando a equação 1:

$$
X c(\%)=\frac{\Delta H m-\Delta H c}{\Delta H m^{\circ}} \times 100 \%
$$

Onde: Xc é a porcentagem de cristalinidade, $\Delta \mathrm{Hm}$ é a entalpia de fusão cristalina da amostra obtida por DSC, $\Delta \mathrm{Hc}$ é a entalpia de cristalização ocorrida durante o aquecimento, neste caso o valor é zero, e o $\Delta \mathrm{Hm}^{0}$ é a entalpia de fusão de equilíbrio para o material teoricamente $100 \%$ cristalino. O valor de $\Delta \mathrm{Hm}^{0}$ para o LDPE é de 293,0 J/g [23]. Para os compósitos, o valor do grau de cristalinidade foi obtido considerando-se a quantidade de fibra de coco presente.

\subsection{Caracterização mecânica dos compósitos LDPE/fibra de coco}

\subsubsection{Ensaios de tração dos compósitos LDPE/fibra de coco}

A Figura 5 apresenta as curvas de tensão por deformação das amostras de LDPE puro e dos compósitos LDPE/fibra de coco através dos resultados obtidos pelo ensaio de tração uniaxial, divididas conforme o teor em massa de fibra de coco, em comparação ao LDPE puro.
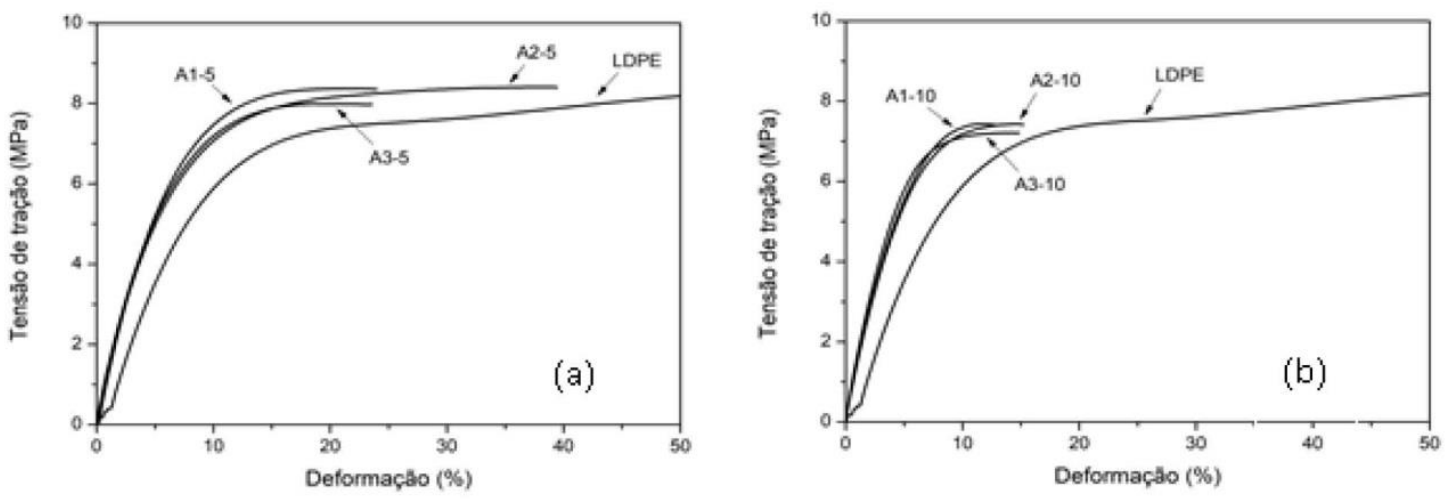

Figura 5: Curvas tensão x deformação das amostras de LDPE puro e dos compósitos (a) sob 5\% m/m de fibra de coco e 
(b) sob $10 \% \mathrm{~m} / \mathrm{m}$ de fibra de coco.

A Tabela 3 apresenta os valores das propriedades mecânicas obtidas pelo ensaio de tração uniaxial das amostras analisadas conforme os diferentes tipos de tratamento aplicados (solução de $\mathrm{NaOH}$ e ultrassom) e os diferentes teores de fibra de coco no compósito polimérico $(5$ e $10 \% \mathrm{~m} / \mathrm{m})$, apresentando a média e os desvios padrão correspondentes dos valores obtidos.

Tabela 3: Propriedades mecânicas obtidas pelo ensaio de tração uniaxial das amostras estudadas.

\begin{tabular}{c|c|c|c|c}
\hline AMOSTRA & $\boldsymbol{\sigma}_{\mathrm{e}}(\mathbf{M P a})$ & $\boldsymbol{\sigma}_{\mathrm{v}}(\mathbf{M P a})$ & $\mathbf{E}(\mathbf{M P a})$ & $\mathbf{A L}(\%)$ \\
\hline $\mathrm{LDPE}$ & $3,10 \pm 0,25$ & $9,42 \pm 0,76$ & $72,86 \pm 13,28$ & $269,77 \pm 51,71$ \\
\hline A1-5 & $2,46 \pm 0,48$ & $8,30 \pm 0,20$ & $154,66 \pm 12,83$ & $39,27 \pm 3,94$ \\
\hline A2-5 & $2,18 \pm 0,05$ & $8,40 \pm 0,14$ & $147,13 \pm 3,07$ & $44,11 \pm 3,12$ \\
\hline A3-5 & $2,44 \pm 0,78$ & $8,02 \pm 0,25$ & $144,82 \pm 23,37$ & $35,92 \pm 5,78$ \\
\hline A1-10 & $2,82 \pm 0,72$ & $7,58 \pm 0,43$ & $159,98 \pm 14,52$ & $18,72 \pm 12,78$ \\
\hline A2-10 & $2,15 \pm 0,13$ & $7,40 \pm 0,16$ & $174,90 \pm 10,44$ & $18,17 \pm 5,44$ \\
\hline A3-10 & $2,55 \pm 1,00$ & $7,10 \pm 0,77$ & $176,55 \pm 21,51$ & $30,32 \pm 8,95$ \\
\hline
\end{tabular}

Onde: $\sigma e:$ é a tensão de escoamento, $\sigma \mathrm{v}$ : tensão máxima verdadeira, E: módulo elástico e AL: alongamento na ruptura. A tensão máxima verdadeira foi obtida através de cálculo utilizando a seção transversal dos corpos de prova após ruptura.

\subsubsection{Ensaio de dureza dos compósitos LDPE/fibra de coco}

A Figura 6 apresenta o gráfico com resultados obtidos pelo ensaio de duzera Shore D das amostras de LDPE puro e dos compósitos poliméricos estudados, conforme os diferentes tipos de tratamento aplicados (solução de $\mathrm{NaOH}$ e ultrassom) e os diferentes teores de fibra de coco no compósito polimérico (5 e $10 \% \mathrm{~m} / \mathrm{m})$, apresentando a média e os desvios padrão correspondentes dos valores obtidos.

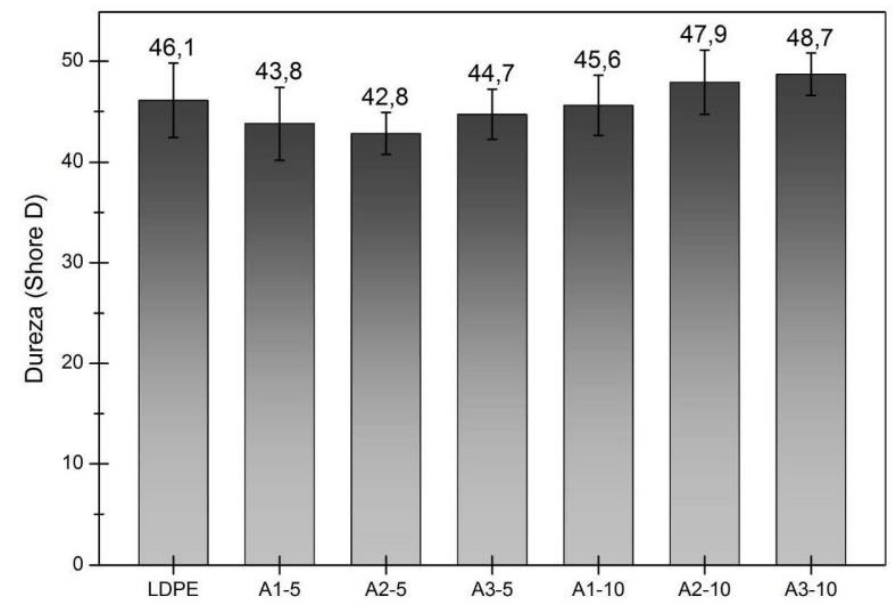

Figura 6: Valores de dureza Shore D das amostras de LDPE puro e dos compósitos LDPE/FC com 5 e $10 \%$ de FC após os diferentes tratamentos.

\subsection{Caracterização morfológica dos compósitos LDPE/fibra de coco}

A Figura 7 apresenta as imagens obtidas por MEV da superfície de fratura criogênica dos compósitos com 5 e $10 \% \mathrm{~m} / \mathrm{m}$ de fibra de coco, com diferentes tratamentos da fibra. 

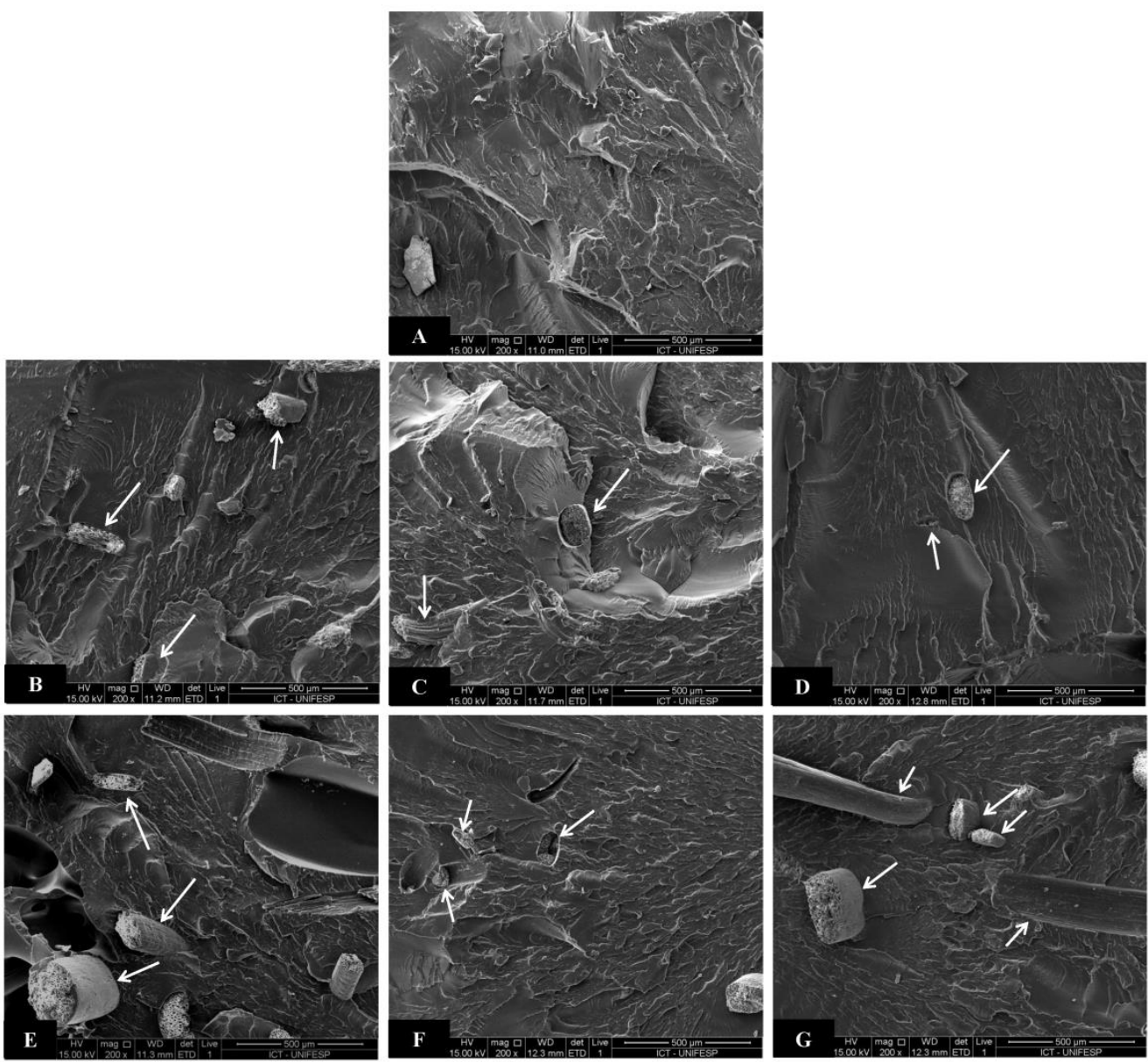

Figura 7: Micrografias de MEV dos compósitos (a) LDPE puro, (b) A1-5 e (c) A2-5, (d) A3-5, (e) A1-10, (f) A2-10 e (G) A3-10, com ampliação de 200x.

A Figura 8 apresenta imagens obtidas por MEV da superfície de fratura criogênica dos compósitos com 5\% m/m de fibra de coco com diferentes tratamentos empregados nas fibras (A1-5, A2-5 e A3-5).
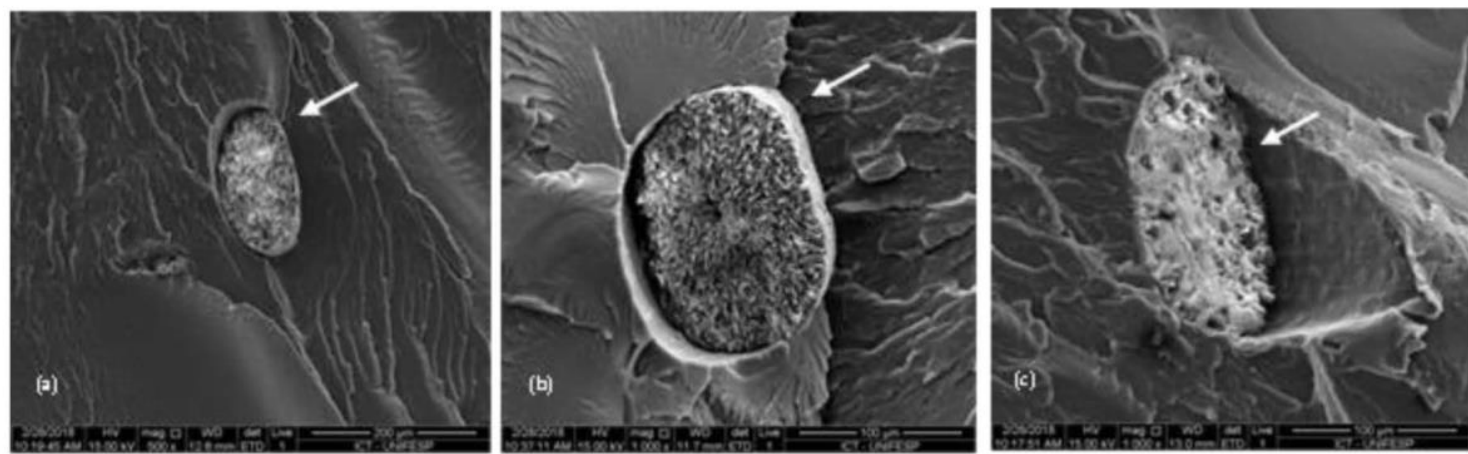

Figura 8: Micrografias de MEV dos compósitos (a) A1-5, (b) A2-5 e (c) A3-5, com ampliação de 1000x. 
A Figura 9 apresenta imagens obtidas por MEV da superfície de fratura dos compósitos com 10\% $\mathrm{m} / \mathrm{m}$ de fibra de coco com diferentes tratamentos empregados nas fibras (A1-10, A2-10 e A3-10).
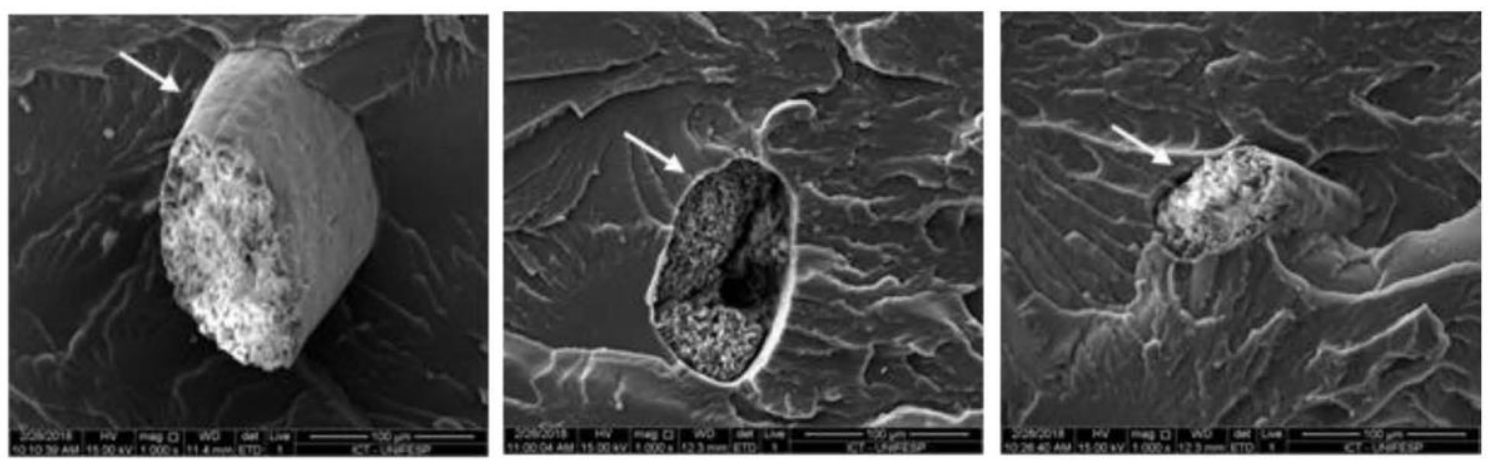

Figura 9: Micrografias de MEV dos compósitos (a) A1-10, (b) A2-10 e (c) A3-10, com ampliação de 1000x.

\subsection{Caracterização de superfície dos compósitos LDPE/fibra de coco}

A Tabela 4 apresenta os valores de ângulo e imagem de contato entre o líquido e as amostras obtidas pelo goniômetro.

Tabela 4: Valores dos ângulos e imagens de contato entre o líquido e as amostras obtidos pelo goniômetro.

\begin{tabular}{c|c|c|}
\hline AMOSTRA & $\begin{array}{c}\text { Ângulo de } \\
\text { contato }\end{array}$ & Imagem \\
\hline LDPE & $90,5 \pm 1,56$ & \\
\hline A1-5 & $84,3 \pm 0,74$ & \\
\hline A2-5 & $86,7 \pm 0,66$ & \\
\hline A3-5 & $86,6 \pm 0,86$ & \\
\hline A2-10 & $81,2 \pm 0,67$ & \\
\hline & & \\
\hline & & \\
\hline
\end{tabular}




\section{DISCUSSÃO}

$\mathrm{O}$ tratamento superficial das fibras foi observado por microscopia eletrônica de varredura (MEV). Por meio das micrografias obtidas na Figura 3, pode-se avaliar a região superficial das fibras de coco após serem submetidas aos tratamentos com solução de $\mathrm{NaOH}$ e ponteira ultrassônica em meio aquoso. Observou-se que não houve diferença significativa na superfície morfológica das fibras modificadas via extração alcalina (Fig. 3-b) quando em comparação com as fibras in natura (Fig. 3-a). No entanto, é possível notar na Figura 3-b que sua superfície permanece rugosa, visto uma possível exposição da celulose e remoção parcial da lignina, hemicelulose e impurezas, efeito gerado pelo tratamento com $\mathrm{NaOH}$, fenômeno observado por Carvalho et al. [24]. Esses componentes em grande quantidade na fibra podem dificultar sua adesão com a matriz polimérica. Uma vez removidos em proporção adequada, reduzem a hidrofilicidade das fibras de coco e facilitam o acoplamento com o LDPE no compósito, como observado nas micrografias das amostras representadas nas Figuras 8 e 9. Na Figura 3-c, da fibra de coco tratada com ponteira ultrassônica em meio aquoso, nota-se uma superfície mais lisa e pouco áspera comparada com a fibra de coco in natura, no entanto, ainda é possível observar a presença de certa rugosidade nas fibras de coco, essa rugosidade superficial é desejável para a adição desse material no compósito pois poderá facilitar o ancoramento das cadeias poliméricas do LDPE na fibra de coco tratada. Com relação aos aspectos morfológicos das fibras, ambos os tratamentos mostraram-se eficazes, visto que não foram observadas em sua superfície regiões com danificações ou fissuras que levassem a algum tipo de quebra e/ou deterioração das fibras de coco.

No que concerne às propriedades térmicas dos compósitos de LDPE/fibras de coco, as temperaturas de fusão cristalina (Tm) no aquecimento e de cristalização (Tc) durante o resfriamento do LDPE no compósito podem ser determinadas a partir dos picos endotérmicos de aquecimento, em torno de $107^{\circ} \mathrm{C}$, e exotérmicos de resfriamento, em torno de $93{ }^{\circ} \mathrm{C}$, respectivamente, para todas as amostras, conforme pode ser observado na Figura 4. Através dos resultados encontrados na Tabela 2, percebe-se que a adição do reforço não apresentou influência significativa nas temperaturas de fusão cristalina no segundo aquecimento e de cristalização e no grau de cristalinidade (Xc) dos compósitos poliméricos, visto que se mantiveram praticamente inalterados quando em comparação com o LDPE puro. A variação de energia entálpica nos compósitos com maior porcentagem em massa de fibras foi ligeiramente menor em relação ao LDPE puro. No entanto, como a diferença foi muito pequena, não é possível assumir de fato que a porcentagem de fibra de coco implicou na diminuição do grau de cristalinidade do polímero. Analisando o comportamento térmico dos compósitos no primeiro aquecimento, nota-se que a adição de fibra de coco in natura modifica o grau de cristalinidade do LDPE, observando-se um aumento de cerca de $18 \%$ no grau de cristalinidade do LDPE com a adição de $10 \%$ $\mathrm{m} / \mathrm{m}$ de fibra de coco. Por outro lado, a adição de mesmo teor de fibra de coco com os diferentes tratamentos apresentaram resultados semelhantes ao LDPE puro, ou seja, as fibras de coco tratadas pouco influenciaram na cristalização do LDPE. No primeiro aquecimento observa-se o efeito do processamento adotado para obtenção dos compósitos, envolvendo os diferentes ciclos térmicos (misturador e prensagem) e condições de resfriamento. De certa forma, observou-se que as condições adotadas para o processamento auxiliaram no aumento do grau de cristalinidade do LDPE com o aumento no teor de fibras de coco in natura, atuando, para esse teor, como um agente nucleante para o LDPE, no entanto, nota-se que existe um teor crítico de fibra de coco para ocorrência desse fenômeno. Já no segundo aquecimento, excluindo-se o histórico térmico sofrido pelo material, nota-se pouca influência da fibra de coco e de seus tratamentos no grau de cristalinidade do LDPE. Esses resultados são importantes, uma vez que a adição da fibra de coco não altera as condições de processamento desses materiais, o que torna viável sua produção na indústria de plástico.

Com relação aos tratamentos superficiais nas fibras de coco, percebe-se que também não houve variação significativa nos valores de Tm, sugerindo que tanto o beneficiamento químico por solução de $\mathrm{NaOH}$ como o tratamento mecânico via ponteira ultrassônica, quando em comparação com as fibras in natura, não afetam a cristalinidade do LDPE. A pequena variação entre os resultados de temperatura de fusão cristalina e do grau de cristalinidade pode ter ocorrido em função de um baixo tempo de reação ou concentração de Na$\mathrm{OH}$ abaixo da ideal - neste trabalho, de $10 \% \mathrm{~m} / \mathrm{m}$. Como observado por Suardana et al. [25], concentrações muito baixas de $\mathrm{NaOH}$ em solução não promovem uma remoção adequada dos componentes da fibra, da mesma forma que concentrações acima da ideal geram o fenômeno da fibrilação - exposição das fibrilas na superfície da fibra, visto que a hemicelulose e a lignina promovem seu agrupamento na estrutura da fibra vegetal.

Por meio dos dados obtidos pelo ensaio de tração uniaxial na Tabela 3, nota-se que os compósitos de LDPE e fibra de coco, com diferentes teores e tratamentos da fibra apresentaram valores inferiores de tensão máxima comparativamente ao LDPE puro. Observou-se ainda que aumentando-se o teor de fibra de coco na composição ocorre consequente diminuição no valor da tensão máxima. O fenômeno pode ser um indicativo da fraca adesão entre a fibra vegetal e a matriz polimérica. Um aumento do teor de fibra torna ainda maior a 
região de interface entre os dois componentes do compósito, que pode reduzir a resistência mecânica sob tração. Quanto maior a fração volumétrica fibrosa, mais difícil com que ocorra uma distribuição homogênea das mesmas pela matriz polimérica, podendo interferir na transferência de tensão da matriz para o reforço fibroso, quando o material for solicitado sob tração. Por outro lado, observa-se um aumento bastante significativo no módulo elástico dos compósitos em comparação ao LDPE puro (Tabela 3), o módulo elástico está relacionado a rigidez do material, a adição de fibra de coco torna o material mais rígido decorrente do esforço que é capaz de suportar. Além disso, fibras de coco possuem um elevado teor de lignina quando em comparação a outros materiais lignocelulósicos, o que reduz a tração média e eleva a rigidez do material, como reportado por Esmeraldo [8]. Nota-se que o tratamento superficial das fibras de coco também está relacionado com a fração mássica das fibras adicionadas no compósito. Para adição de $5 \% \mathrm{~m} / \mathrm{m}$ de fibra de coco nota-se aumento do módulo elástico em relação ao LDPE puro, para todas as composições, porém o efeito do tratamento superficial não é evidenciado. No entanto, a adição de $10 \% \mathrm{~m} / \mathrm{m}$ de fibra de coco tratadas aumentam cerca de $10 \%$ o módulo em relação ao compósito com mesmo teor de fibra de coco in natura e cerca de $140 \%$ em relação ao LDPE puro, sendo muito significativo.

Conforme discutido, uma concentração não adequada de $\mathrm{NaOH}$ em solução pode influenciar nas propriedades mecânicas finais dos compósitos. De acordo com Skreekala [26], baixas concentrações de $\mathrm{NaOH}$ impossibilitam a remoção da lignina da superfície das fibras de coco em proporção adequada e podem, dessa forma, também reduzir o diâmetro das mesmas, levando a uma diminuição da resistência máxima de tração da fibra que pode ocasionar em menor reforçamento para os compósitos. Ao analisar o efeito da extração alcalina em LDPE reforçado com fibra de bagaço de cana-de-açúcar, Muller et al. [27] obtiveram resultado semelhante ao obtido nesse trabalho, notando que houve uma pequena redução no valor médio de tensão máxima nos compósitos reforçados pela fibra tratada, o que provavelmente ocorreu por conta de uma eventual degradação da fibra. É importante ressaltar a influência da anisotropia nas propriedades finais do compósito. Se as fibras estão dispostas de forma aleatória pela matriz polimérica, como neste trabalho em decorrência do processamento utilizado, a rigidez e o módulo elástico tendem a apresentar valores mais baixos do que se distribuídas paralelamente umas às outras e ao sentido de aplicação da força.

A taxa de deformação do LDPE puro foi superior a $273 \%$, identificando o perfil característico de materiais plásticos, a uma deformação elástica inicial seguida de escoamento além da deformação plástica. Ao adiconar as fibras, houve uma redução da ductilidade das amostras, considerando-se uma menor taxa de deformação dos compósitos até a tensão máxima em tração (conforme visto na Tabela 3), decorrente de uma maior quantidade de reforço distribuído pela matriz polimérica.

Através dos ensaios de dureza, conforme observado na Figura 6, é possível perceber que a adição das fibras modificou levemente o comportamento mecânico de dureza dos compósitos. Quando em comparação com as amostras de LDPE puro, nota-se que houve uma redução na dureza dos compósitos a $5 \% \mathrm{~m} / \mathrm{m}$ de fibra de coco, e um leve aumento na dureza quando a $10 \% \mathrm{~m} / \mathrm{m}$ de fibra de coco. Assim como nos resultados dos ensaios sob tração, os diferentes tratamentos mostraram resultados muito próximos entre si, porém, conforme discutido anteriormente, sob $10 \% \mathrm{~m} / \mathrm{m}$ de fibra de coco é possível notar um aumento mais acentuado do módulo elástico do material, fenômeno que também é observado no ensaio de dureza, com valores superiores aos compósitos reforçados pelas fibras in natura. Sugere-se assim que o efeito dos tratamentos nas fibras de coco foi notado com maior evidência e exerceu maior influência na presença de um maior teor de fibra de coco.

O efeito da aplicação dos tratamentos das fibras de coco na região interfacial pode ser observado nas Figuras 7 a 9, que representam a micrografia da fratura criogênica da superfície morfológica das amostras. Em todos os compósitos percebe-se uma interface bem definida entre os componentes (ver setas destacadas nas micrografias), sugerindo que a matriz de LDPE teve um bom acoplamento com a fibra natural e modificada, além de uma boa dispersão da fibra na matriz. Assim, nota-se que os tratamentos utilizados melhoram a adesão entre os componentes do compósito polimérico.

O grau de molhabilidade, ou seja, a capacidade que um líquido tem de se espalhar sobre a superfície de um material, pode ser determinado pelos testes de ângulo de contato encontrados na Tabela 4. É sabido que quanto menor o ângulo de contato formado entre a gota do líquido e o material onde o mesmo é despejado, maior a capacidade de molhamento, caracterizando uma boa interação entre a fibra e a matriz do compósito polimérico. Assim, os resultados se mostraram eficientes, visto que para todas as amostras dos compósitos o ângulo de contato foi menor quando em comparação ao LDPE puro, de caráter hidrofóbico. Nota-se, assim, um aumento no grau de hidrofilicidade do material. Da mesma forma, houve uma redução ainda maior do ângulo de contato e um aumento do teor hidrofílico para as amostras com 10\% de fibra de coco em massa, levando-se em consideração que a adição das fibras, de caráter hidrofílico, reduz o teor hidrofóbico do LDPE. 
Analisando-se os dados encontrados pelos testes do ângulo de contato e a interface dos compósitos nas micrografias nas Figuras 8 e 9, nota-se uma região interfacial mais bem definida nas amostras sob tratamento via extração alcalina (Fig. 8-b e 9-b), e ângulos de contato ligeiramente menores nos compósitos reforçados por $10 \% \mathrm{~m} / \mathrm{m}$ de fibra de coco, sob o mesmo tratamento, sugerindo que o tratamento químico por solução de $\mathrm{NaOH}$ se mostrou relativamente mais eficiente em relação aos demais. Favoreceu-se um caráter mais hidrofóbico pela redução da polaridade da fibra através da reação com os grupos - $\mathrm{OH}$ terminais e aumentando a molhabilidade pela matriz polimérica.

Considerando-se que como neste trabalho as fibras de coco foram dispostas de maneira aleatória pela matriz polimérica, vale ressaltar que pode haver certa variação entre os ângulos de contato encontrados para uma mesma amostra, em função da região de aplicação da gota na superfície do compósito.

\section{CONCLUSÕES}

Foram preparados com sucesso compósitos de LDPE reforçados por fibras de coco utilizando uma rota alternativa de processamento através de um homogeneizador termocinético, de forma a avaliar o efeito dos tratamentos superficiais de extração alcalina e ultrassom em meio aquoso nas fibras de coco.

Quanto aos aspectos morfológicos, houve um bom acoplamento entre a fibra e a matriz, e uma boa dispersão e distribuição das fibras de coco pela matriz polimérica para todos os compósitos LDPE/fibra de coco, conforme observado nas micrografias. Os resultados através dos testes de ângulo de contato sugerem uma redução no teor hidrofóbico do LDPE, bem como uma melhor molhabilidade dos compósitos, o que facilita adesão entre LDPE e a fibra de coco. Em ambos os ensaios o tratamento via extração alcalina apresentou-se ligeiramente mais eficiente que os demais casos.

Os ensaios de DSC sugeriram que não houve influência da adição das fibras de coco no comportamento térmico dos compósitos, visto que as temperaturas de fusão cristalina e de cristalização e o grau de cristalinidade se mantiveram próximos para todas as amostras.

Tendo em vista a proposta deste trabalho, ao tratar do desenvolvimento de novas tecnologias verdes que reduzam os impactos ambientais, os resultados encontrados se mostraram bastante promissores para o avanço dessa linha de pesquisa, associados à grande disponibilidade com que fibras naturais são encontradas no território nacional e à necessidade cada vez maior do mundo atual de se adaptar a fontes alternativas mais sustentáveis e ecologicamente viáveis.

\section{AGRADECIMENTOS}

Os autores agradecem à FAPESP (2014/04900-9 e 2016/19978-9) e CNPq (442081/2014-6) pelo suporte financeiro, e à empresa Coco Verde/RJ pelo fornecimento das fibras de coco.

\section{BIBLIOGRAFIA}

[1] PIRES, J. S. C. "Fibras Naturais: Características químicas e potenciais aplicações", Dissertação de Graduação em Ciências Biológicas, Instituto de Biociências de Botucatu, Universidade Estadual Paulista Júlio de Mesquita Filho (UNESP), Botucatu, 2009.

[2] BEDIN, M. G. "Compósitos com polietileno de baixa densidade e fibra de coco in natura e modificada", Dissertação de Mestrado em Engenharia e Ciência de Materiais, Centro de Ciência e Tecnologia, Universidade Estadual do Norte Fluminense Darcy Ribeiro, Rio de Janeiro, 2014.

[3] RAMÍREZ, M. G. L., SATYANARAYANA, K. G., IWAKIRI, S., et al., "Study of the properties of biocomposites. Part I. Cassava starch-green coir fibers from Brazil”, Carbohydrate Polymers, v. 86, n. 4. pp. 1712-1722, 2011.

[4] BENINI, K. C. C. C. "Desenvolvimento e caracterização de compósitos poliméricos reforçados com fibras lignocelulósicas: HIPS/fibra da casca do coco verde e bagaço de cana de açúcar”, Dissertação de Mestrado em Engenharia Mecânica, Faculdade de Engenharia de Guaratinguetá, Universidade Estadual Paulista Júlio de Mesquita Filho (UNESP), Guaratinguetá, 2011.

[5] CASTILHOS, L. F. F., Serviço Brasileiro de Respostas Técnicas, Dossiê técnico: aproveitamento da fibra de coco, Instituto de Tecnologia do Paraná - TECPAR, pp. 3-8, 2011.

[6] BENINI, K. C. C. G. "Inclusão de materiais lignocelulósicos na produção de compósitos plásticos", Dissertação de Pós-Graduação em Ciência e Tecnologia da Madeira, Universidade Federal de Lavras, Lavras, 2013. 
[7] Coco verde - Ciclo do coco. Disponível em: <Disponível em: https://ciclodococo.wordpress.com/justificativas/>. Acesso em: 13 nov 2018.

[8] ESMERALDO, M. A. Preparação de novos compósitos suportados em matriz de fibra vegetal/natural, Dissertação de Mestrado em Química Inorgânica, Universidade Federal do Ceará, Fortaleza, 2006.

[9] CLAUS, C. Ciência das fibras: coco. Disponível em: <Disponível em: https://pt.scribd.com/doc/38712098/Fibra-do-coco >. Acesso em: 02 set 2018.

[10] PEREIRA, C. L. "Aproveitamento do resíduo do coco verde para produção de compósitos destinados à construção rural", Tese de Doutorado em Zootecnia, Faculdade de Zootecnia e Engenharia de Alimentos, Universidade de São Paulo (USP), Pirassununga, 2012.

[11] FURTADO, C. F. C., GADELHA, M. A. C., "Os impactos ambientais do consumo do coco verde na Praia do Futuro em Fortaleza - CE”, Faculdade Integrada da Grande Fortaleza, Fortaleza, 2012.

[12] EMBRAPA. Empresa Brasileira de Pesquisa Agropecuária. Beneficiamento da casca de coco verde. Disponível em: <Disponível em: https://www.cnpat.embrapa.br/home/portfolio/tecnologia.php?id=10 >. Acesso em 02 set 2018.

[13] COUTINHO, F. M. B., MELLO, I. L., SANTA MARIA, L. C., "Polietileno: principais tipos, propriedades e aplicações", Polímeros, São Carlos, v. 13, n. 1, 2013.

[14] AMUTHAKKANNAN, V., MANIKANDAN, J. T., WINOWLIN JAPPES, M., et al., "Effect of fibre length and fiber content on mechanical properties of short basalt fibre reinforced polymer matrix composites", Materials Physics and Mechanics 16, Institute of Problems of Mechanical Engineering, Russia, pp. 107-117, 2013.

[15] SAHEB, D. N., JOG, J. P., "Natural Fiber Polymer Composites: A Review", Advances in Polymer Technology, v. 18, n. 4, pp. 351-360, 1999.

[16] LI, X., TABIL, L. G., PANIGRAHI, S., "Chemical treatments of natural fiber for use in natural fiberreinforced composites. A review", Journal of Polymers and the Environment. 2007. v. 15, n. 1, pp. 25-33. 2007.

[17] FARIA, J. G., CAVALCANTE, R. C., CANABARRO, B. R., et al., "Surface Lignin Removal on Coir Fibers by Plasma Treatment for Improved Adhesion in Thermoplastic Starch Composites", Carbohydrate Polymers, 2017.

[18] PEREIRA, A. L. S. "Extração de nanocelulose de fibras vegetais", Dissertação de Graduação em Engenharia Química, Universidade Federal do Ceará, Fortaleza, 2010.

[19] NAM, T. H., OGIHARA, S., TUNG, N. H., KOBAYASHI, S., "Effect of alkali treatment on interfacial and mechanical properties of coir fiber reinforced poly(butylene succinate) biodegradable composites", Composites: Part B 42, v. 42, n. 6, pp. 1648-1656, 2011.

[20] HUSSAIN, S. A., "Mechanical properties of green coconut fiber reinforced HDPE polymer composite", International Journal of Engineering Science and Technology (IJEST), v. 3, n. 11, pp. 7942-7952, 2011.

[21] HAGENSON, C. L., DORAISWAMY L. K., "Comparison of the effects of ultrasound and mechanical agitation on a reacting solid-liquid system", Chemical Engineering Science, Great Britain, v. 53, n. 1, pp. 131-148, 1998.

[22] SANTOS, P. A., SPINACÉ, M. A. S., FERMOSELLI, K. K. G., et al., "Efeito da forma de processamento e do tratamento da fibra de curauá nas propriedades de compósitos de Poliamida-6." Polímeros: Ciência e Tecnologia, v. 19, n. 1, pp. 31-39, 2009.

[23] WILfONG, D. L., KNIGHT, G. W., "Journal of Polymer Science: Part B”, Polymer Physics, v. 28, p. $861,1990$.

[24] CARVALHO, K. C. C., MULiNARI, D. R., VOORWALD, H. J. C., et al., "Chemical modification effect on the mechanical properties of HIPS/coconut fiber composites", BioResources, v. 5, n. 2, pp. 1143$1155,2010$.

[25] SUARDANA, N. P. G., PIAO, Y., LIM, J. K., "Mechanical properties of hemp fibers and hemp/PP composites: effects of chemical surface treatment", Materials Physics and Mechanics, v. 11, pp. 1-8, 2011.

[26] SKREEKALA, M., KUMARAN, G., JOSEPH, S., et al., "Oil palm fiber reinforced phenol formaldehyde composites: influence of fiber surface modification on the mechanical performance", Applied Composite Materials, v. 7, n. 5, pp. 295-329, 2000. 
[27] MULLER, R. L., MAHLMANN, C. M., RODRIGUEZ, A. L., et al., "Compósito de polietileno de baixa densidade e fibra de bagaço de cana-de-açúcar", Anais 9 ${ }^{\circ}$ Congresso Brasileiro de Polímeros, 2007.

\section{ORCID}

Yves Nicolau Wearn

https://orcid.org/0000-0002-8542-8254

Larissa Stieven Montagna

https://orcid.org/0000-0002-7947-3112

Fábio Roberto Passador

https://orcid.org/0000-0001-5239-5962 